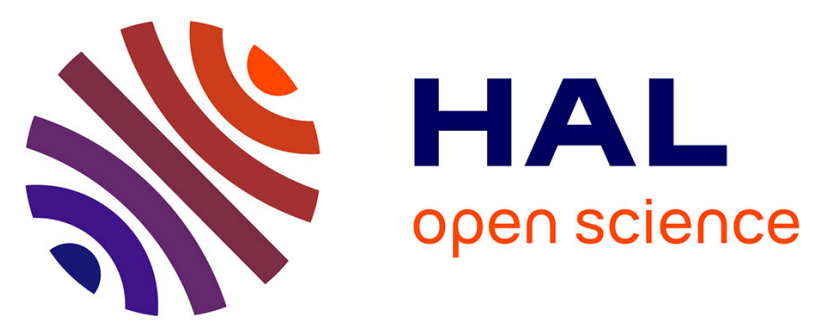

\title{
Generation of parabolic pulses and applications for optical telecommunications
}

Christophe Finot, John M. Dudley, D.J. Richardson, Guy Millot

\section{To cite this version:}

Christophe Finot, John M. Dudley, D.J. Richardson, Guy Millot. Generation of parabolic pulses and applications for optical telecommunications. 11th International Conference on Transparent Optical Network, Jun 2009, Ponta Delgada, Portugal. pp.Tu.D1.5, 10.1109/ICTON.2009.5185133 . hal00470022

\section{HAL Id: hal-00470022 \\ https://hal.science/hal-00470022}

Submitted on 29 Jul 2010

HAL is a multi-disciplinary open access archive for the deposit and dissemination of scientific research documents, whether they are published or not. The documents may come from teaching and research institutions in France or abroad, or from public or private research centers.
L'archive ouverte pluridisciplinaire HAL, est destinée au dépôt et à la diffusion de documents scientifiques de niveau recherche, publiés ou non, émanant des établissements d'enseignement et de recherche français ou étrangers, des laboratoires publics ou privés. 


\title{
Generation of parabolic pulses and applications for optical telecommunications
}

\author{
Christophe Finot ${ }^{1}$, John M. Dudley ${ }^{2}$, David J. Richardson ${ }^{3}$, and Guy Millot ${ }^{1}$ \\ ${ }^{1}$ Institut Carnot de Bourgogne, UMR 5209 CNRS-Université de Bourgogne, 9 Av. A. Savary, Dijon, France \\ ${ }^{2}$ Institut FEMTO-ST, UMR 6174 CNRS-Université de Franche-Comté, Besançon, France \\ ${ }^{3}$ Optoelectronics Research Centre, Southampton University, Southampton, SO17 1BJ, United-Kingdom
}

Tel: 33 (0)380 3959 26,e-mail: Christophe.Finot@u-bourgogne.fr

\begin{abstract}
Parabolic pulses in optical fibers have stimulated an increasing number of applications. We review here the physics underlying the generation of such self-similar pulses as well as the results obtained in a wide-range of passive or active experimental configurations.
\end{abstract}

Keywords: nonlinear optics, fiber amplifiers, optical pulse processing techniques

\section{INTRODUCTION}

Parabolic pulse generation and propagation in optical fibers have motivated many studies in recent years [1]. Specifically, it has been shown that any pulse propagating in a normally dispersive fiber amplifier progressively reshapes into a pulse having a parabolic intensity profile combined with a perfectly linear chirp [2]. This new class of optical pulse is often referred to as a similariton pulse, and exhibits several fundamental features that are clearly attractive for applications ranging from high-power ultrashort pulse generation to optical nonlinear processing of telecommunication signals.

We first review the context of similariton pulse formation as well as different experimental techniques that have been proposed to generate a parabolic intensity profile in both amplifying fibers as well as passive fibers. We then discuss some of the numerous applications that can benefit from the intrinsic properties of these pulses.

\section{SELF SIMILAR PARABOLIC PULSES}

During propagation in a fiber amplifier, gain, dispersion and nonlinearity interact together to strongly modify the properties of the initial ultrashort pulses. The resulting longitudinal evolution of the complex electric field envelope $\psi(z, T)$ can be modeled in terms of the non-linear Schrödinger equation (NLSE) with a constant gain :

$$
i \frac{\partial \psi}{\partial z}=\frac{\beta_{2}}{2} \frac{\partial^{2} \psi}{\partial T^{2}}-\gamma|\psi|^{2} \psi+i \frac{g}{2} \psi,
$$

with $\beta_{2}, \gamma$ and $g$ the second order dispersion, the Kerr nonlinear coefficient and the gain, respectively.

With this description, the self-similar dynamics of any pulse evolving in a normally dispersive amplifier is expected to be accurately described by the asymptotic solution found in the limit $z \rightarrow \infty$. The asymptotic complex field is for $|T|<T_{p}(z)$ given by:

$$
\psi(z, T)=A_{o} \exp \left(\frac{g z}{3}\right) \sqrt{1-\left(\frac{T}{T_{p}}\right)^{2}} \exp \left[i\left(\frac{3 \gamma A_{o}^{2}}{2 g} \exp \left(\frac{2 g z}{3}\right)-\frac{g}{6 \beta_{2}} T^{2}\right)\right]
$$

with $\psi(z, T)=0$ for $|T|>T_{p}(z)$ [2]. Here, $A_{o}$ is a constant amplitude term $A_{o}=1 / 2\left(g U_{i n} / \sqrt{\gamma \beta_{2} / 2}\right)^{1 / 3}$ where $U_{i n}$ is the initial pulse energy. This solution corresponds to a compactly-supported pulse with a parabolic intensity profile whose zero-crossing points are given by $T_{p}(z)=\left(6 A_{0} / g\right) \sqrt{\gamma \beta_{2} / 2} \exp (g z / 3)$. These parabolic pulses have a peak power given by $P_{o}(z)=A_{o}^{2} \exp (2 g z / 3)$ and a linear chirp with slope $C=g / 6 \pi \beta_{2}$. In the frequency domain, the corresponding asymptotic power spectrum is also parabolic with zero-crossing points of $\pm v_{p}(z)$ where $v_{p}(z)=\left(A_{0} / 2 \pi\right) \sqrt{2 \gamma / \beta_{2}} \exp (g z / 3)$.

From those analytical expressions, we identify several important features. First, once acquired, the parabolic shape is maintained as the pulse experiences exponential temporal and spectral broadening as well as an amplitude increase. In other words, the similariton is able to resist the deleterious effects of the wave-breaking $[1,2]$, a phenomenon which usually seriously degrades the evolution of a pulse evolving in a nonlinear normally dispersive medium. More remarkably, the output characteristics depend only on the energy of the initial pulse and are independent of its intensity profile: the asymptotic state actually behaves as an attractor of the system [1, 3]. In other words, initial pulses with significantly different temporal widths but with the same energy will converge after a sufficient propagation length in the amplifier towards the analytical state predicted by Eq. (2). 
The dynamics of a pair of optical similaritons with the same or different central wavelengths has also been theoretically and experimentally investigated : similaritons are robust against collisions, whereas the interaction of similaritons leads to the generation of high-repetition rate dark soliton trains [4].

\section{EXPERIMENTAL GENERATION}

The first clear experimental demonstration of the theoretical predictions of Eq. (2) have been carried out in the framework of ytterbium-doped fiber amplifiers [2]. A precise intensity and phase measurement based on the frequency resolved optical gating (FROG) definitively confirmed the parabolic intensity profile of the emerging picosecond pulses, as well as the high linearity of the chirp. Since then, several other experiments have benefited of the parabolic features in ytterbium doped fibers with increasing output power levels and various initial femtosecond seeds from passively modelocked fiber lasers with repetition rates of a few tens of $\mathrm{MHz}$ [2] to VECSELs with GHz repetition rates [5]. Since the pulse reshaping process is not restricted to a specific rareearth dopant, generation in erbium-doped amplifiers at telecommunication wavelengths has also been successfully tested $[1,6]$.

(a)

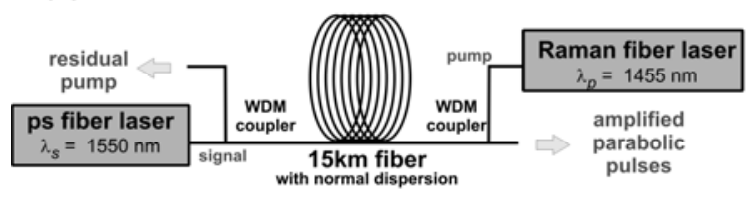

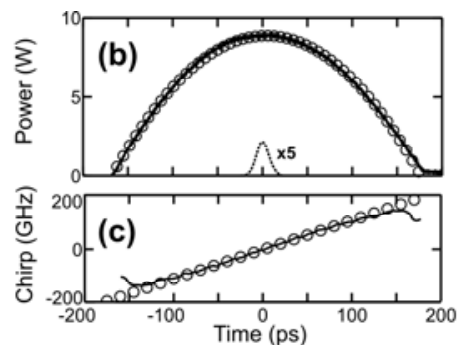

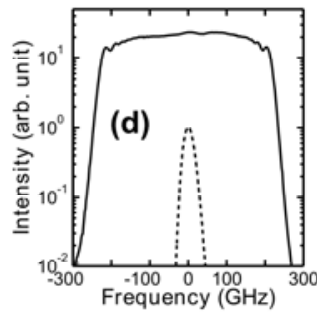

Figure 1. Generation of parabolic pulses through Raman amplification at telecommunication wavelength

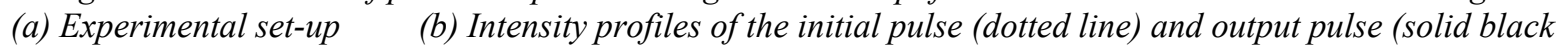
line) compared with a parabolic fit (circles) $\quad$ (c) Chirp of the output pulse compared with a linear fit (circles) (d) Spectrum of the initial and output pulses (dashed and solid lines respectively). Results adapted from [7].

Other gain mechanisms are also fully suitable for asymptotic parabolic generation and configurations based on the use of the Raman amplification effect have been successfully investigated [7]. As an illustration, Fig. 1 presents the set-up and results of an experiment where similaritons are generated through Raman amplification at telecommunication wavelengths. In this all-fiber set-up using only standard devices, initial 14-ps pulses are adiabatically amplified in a 15-km normally dispersive fiber and the output pulse intensity profile is recorded in real-time by a high-speed photodetector and oscilloscope. The emerging 200-ps pulses clearly exhibit the expected parabolic intensity profile combined with a highly linear chirp. The output optical spectrum also highlights the clear reshaping of the pulse, with a low ripple in the central part and a rapid decrease of the wings.

The generation of parabolic pulses was first outlined in the context of an active amplifying medium. Nevertheless, it is also of interest to propose alternative generation methods, especially in the context of optical telecommunications. Several specific techniques have been recently experimentally demonstrated. Based on the observation that a longitudinal decrease of the normal dispersion is formally equivalent to optical gain, it has for example been proposed to use continuously tapered dispersion decreasing [8, 9] or comb-like fibers [10]. For a given set of input parameters, it has been shown that only two carefully chosen normally dispersive optical fiber segments were required [11-13] (Fig. 2). It is also possible to achieve passive linear pulse shaping based on superstructured fiber Bragg gratings, [14-16] or arrayed waveguide gratings [17].

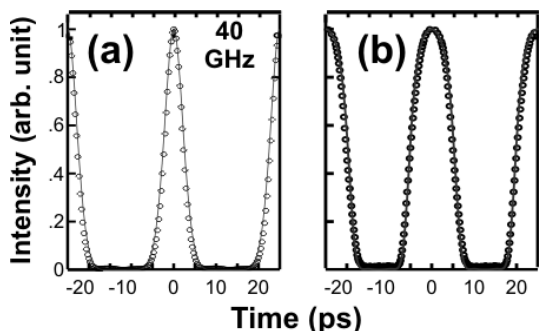

O experimental FROG results

numerical simulations based on NLSE

... first order Bessel function of the first kind

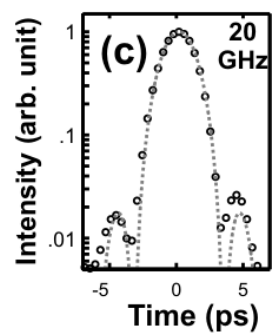

Figure 2. Nonlinear passive reshaping observed in a two segment configuration. Initial Gaussian pulse train (a) is reshaped into parabolic pulse train (b) after propagation in a normal dispersion shifted fiber followed by a highly nonlinear fiber. (c) Details of parabolic pulse after linear compression Results adapted from [12].

\section{APPLICATIONS}

Optical similaritons are not only of theoretical interest but they have very wide practical potential and have stimulated numerous applications both in ultrashort high-power pulse generation and in optical pulse processing. 
The field of ultrashort high-power pulse generation has strongly benefited from the highly linear chirp of the amplified pulses $[2,5]$. Indeed, the direct nonlinear amplification of pulses avoids the necessity of a prechirping stage as required in the linear chirped pulse amplification approach where the ultimate goal is to avoid any nonlinearity by stretching the initial pulse and therefore reducing the initial peak power. The approach based on parabolic amplification is intrinsically different, as it takes advantage of the nonlinear optical spectrum expansion of the pulse. Thanks to the high level of linearity of this chirp, it is possible to efficiently compensate for the strong frequency chirp by using a pair of diffraction gratings so that high quality recompressed pulses can be obtained with final temporal durations much shorter than the initial seed pulse. Indeed, the theoretical pulse shape obtained after compression of a perfectly parabolic pulse is a first order Bessel function of the first kind that exhibits noticeably low sidelobes (see Fig. 2(c)). Several experimental works have reported high performance results, with temporal widths below $100 \mathrm{fs}$ and peak-powers exceeding $4 \mathrm{MW}$ [18] or pulse energy at the microjoule level [19]. Parabolic amplification can also sustain GHz repetition rates [5, 6] and can be integrated in an all-fiber set-up with the judicious use of hollow-core photonic bandgap fibers employed as compression stages [5]. Additional nonlinear higher-order soliton compression has enabled the generation of pulses with temporal durations as short as $20 \mathrm{fs}$ (i.e. four optical cycles at $1550 \mathrm{~nm}$ ) [1].
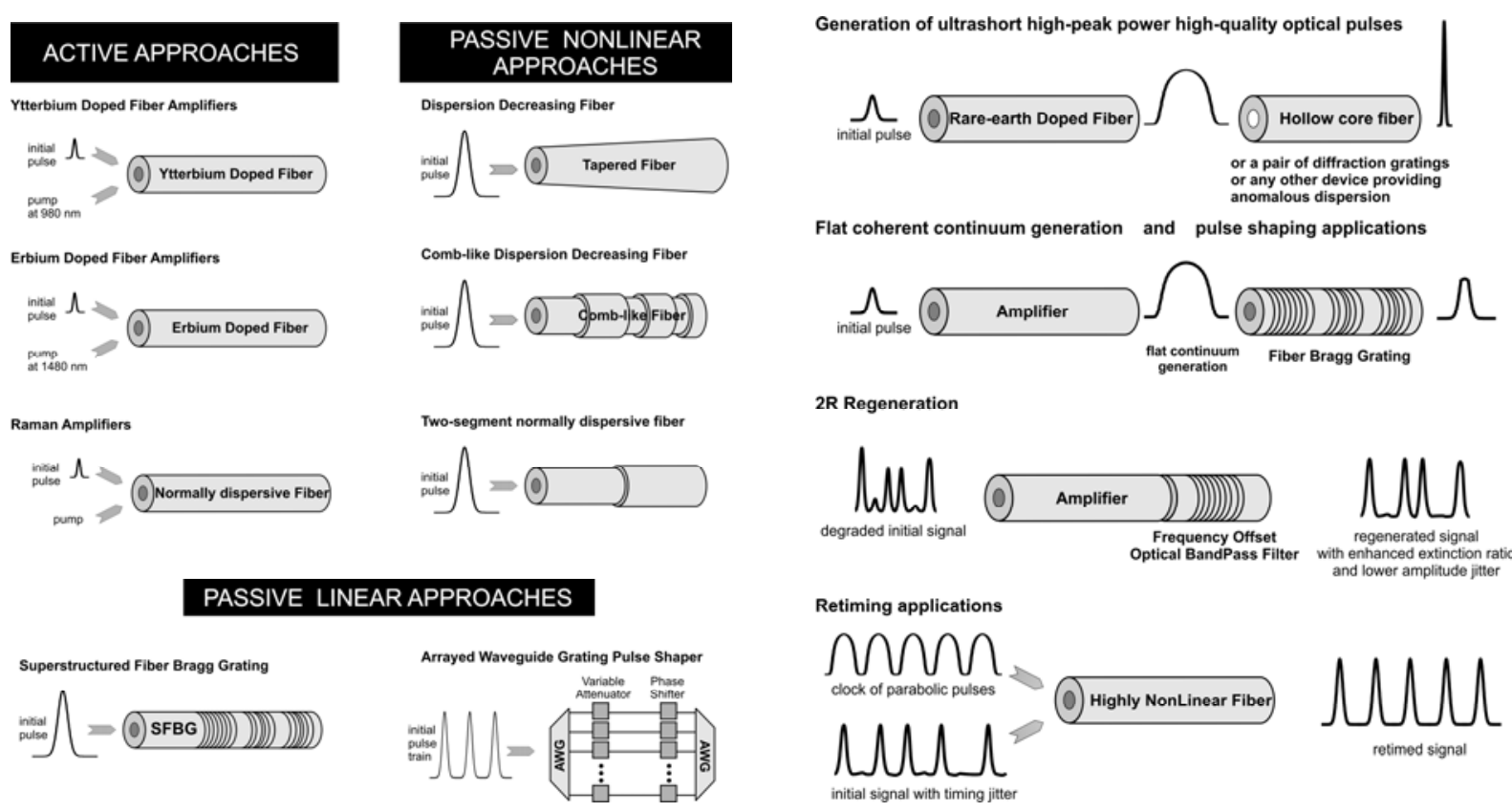

Figure 3. Various approaches for parabolic pulse generation and potential applications

Highly coherent pulse generation has also taken advantage of the parabolic pulses ability to resist the effects of optical wave-breaking. Continuum with low spectral ripple and high energy spectral density in the central part can indeed be achieved [14] and the temporal coherence of the continuum obtained in the normal dispersion regime has been found superior to the case of continuum generated in the anomalous regime. Such features can be of great interest for applications such as pulse shaping [20] or multiwavelength picosecond sources. Examples of low-noise multichannel sources running at $10 \mathrm{GHz}$ and covering the whole C-band of the optical telecommunications have therefore been demonstrated based on km-long erbium doped fiber amplifier [6] or on the passive propagation of a parabolic pulse in a highly non-linear fiber [14].

In the high-bit rate telecommunication context, using parabolic pulses has also enabled noticeable improvements of existing optical pulse processing techniques based on the quasi-instantaneous Kerr response of highly nonlinear optical fibers. Various applications targeting the optical regeneration of an optical signal impaired by amplitude or timing jitter have been proposed. Distributed Raman amplification in a normally dispersive fiber followed by offset spectral filtering has for example been shown to improve the ability of a $2 \mathrm{R}$ regenerator to eliminate spurious noise pulses as well as to simultaneously reduce any fluctuations in the signal ' 1 ' bit level [21]. Taking advantage of the perfectly linear chirp that can be generated through cross-phase modulation (XPM) induced by a parabolic pulse, it is possible to increase the level of tolerable temporal jitter that an optical retiming system can accommodate [15]. More recently, parabolic phase profiles induced by XPM have also been shown to be beneficial for the restoration of the intensity profile of pulses degraded by linear dispersive effects. Indeed, the time-domain optical Fourier transformation approach is based on imparting a highly linear chirp to the distorted data bits and the use of parabolic pulses represents an attractive solution to generate the required chirp for picosecond pulse signals [16, 17]. 


\section{CONCLUSIONS}

In conclusion, parabolic self-similar pulses represent a new class of ultrashort optical pulses that have opened new avenues of fundamental and applied photonics research. A wide set of active or passive techniques has progressively become available to generate these pulses for applications such as ultrashort high-power pulse generation, highly coherent continuum sources and optical signal processing in high-bit rate telecommunications, to name just a few.

\section{ACKNOWLEDGEMENTS}

We would like to acknowledge the significant scientific contributions of B. Kibler, S. Pitois, J. Fatome, K. Hammani, S. Wabnitz (ICB), P. Dupriez, F. Parmigiani, L. Provost, M. Ibsen, P. Petropoulos (ORC).

\section{REFERENCES}

[1] J. M. Dudley, C. Finot, G. Millot, and D. J. Richardson, "Self-similarity in ultrafast nonlinear optics," Nat. Phys., vol. 3, pp. 597-603, 2007.

[2] M. E. Fermann, V. I. Kruglov, B. C. Thomsen, J. M. Dudley, and J. D. Harvey, "Self-similar propagation and amplification of parabolic pulses in optical fibers," Phys. Rev. Lett., vol. 84, pp. 6010-6013, 2000.

[3] C. Finot, G. Millot, and J. M. Dudley, "Asymptotic characteristics of parabolic similariton pulses in optical fiber amplifiers," Opt. Lett., vol. 29, pp. 2533-2535, 2004.

[4] C. Finot and G. Millot, "Interactions of optical similaritons," Opt. Express, vol. 13, pp. 5825-5830, 2005.

[5] P. Dupriez, C. Finot, A. Malinowski, J. K. Sahu, J. Nilsson, D. J. Richardson, K. G. Wilcox, H. D. Foreman, and A. C. Tropper, "High-power, high repetition rate picosecond and femtosecond sources based on Yb-doped fiber amplification of VECSELS," Opt. Express, vol. 14, pp. 9611-9616, 2006.

[6] Y. Ozeki, Y. Takushima, K. Aiso, and K. Kikuchi, "High repetition-rate similariton generation in normal dispersion erbium-doped fiber amplifiers and its application to multi-wavelength light sources," IEICE Trans. Electron., vol. 88, pp. 904-911, 2005.

[7] K. Hammani, C. Finot, S. Pitois, J. Fatome, and G. Millot, "Real time measurement of long parabolic optical similaritons," Electron. Lett., vol. 44, pp. 1239-1240, 2008.

[8] T. Hirooka and M. Nakazawa, "Parabolic pulse generation by use of a dispersion-decreasing fiber with normal group-velocity dispersion," Opt. Lett., vol. 29, pp. 498-500, 2004.

[9] C. Finot, B. Barviau, G. Millot, A. Guryanov, A. Sysoliatin, and S. Wabnitz, "Parabolic pulse generation with active or passive dispersion decreasing optical fibers," Opt. Express, vol. 15, pp. 15824-15835, 2007.

[10] B. Kibler, C. Billet, P. A. Lacourt, R. Ferrière, L. Larger, and J. M. Dudley, "Parabolic pulse generation in comb-like profiled dispersion decreasing fibre," Electron. Lett., vol. 42, pp. 965-966 2006.

[11] C. Finot, L. Provost, P. Petropoulos, and D. J. Richardson, "Parabolic pulse generation through passive nonlinear pulse reshaping in a normally dispersive two segment fiber device," Opt. Express, vol. 15, pp. 852-864, 2007.

[12] C. Finot, J. Fatome, S. Pitois, and G. Millot, "All-Fibered High-Quality Low Duty-Cycle 20-GHz and 40GHz Picosecond Pulse Sources," IEEE Photon. Technol. Lett., vol. 19, pp. 1711-1713, 2007.

[13] S. Boscolo, A. I. Latkin, and S. K. Turitsyn, "Passive nonlinear pulse shaping in normally dispersive fiber systems," IEEE J. Quantum Electron., vol. 44, pp. 1196-1203, 2008.

[14] F. Parmigiani, C. Finot, K. Mukasa, M. Ibsen, M. A. F. Roelens, P. Petropoulos, and D. J. Richardson, "Ultra-flat SPM-broadened spectra in a highly nonlinear fiber using parabolic pulses formed in a fiber Bragg grating," Opt. Express, vol. 14, pp. 7617-7622, 2006.

[15] F. Parmigiani, P. Petropoulos, M. Ibsen, and D. J. Richardson, "Pulse retiming based on XPM using parabolic pulses formed in a fiber Bragg grating," IEEE Photon. Technol. Lett., vol. 18, pp. 829-831, 2006.

[16] T. T. Ng, F. Parmigiani, M. Ibsen, Z. Zhang, P. Petropoulos, and D. J. Richardson, "Compensation of linear distorsions by using XPM with parabolic pulses as a time lens," IEEE Photon. Technol. Lett., vol. 20, pp. 1097-1099, 2008.

[17] T. Hirooka and M. Nakazawa, "All-optical 40-GHz Time-Domain Fourier Transformation using XPM with a dark parabolic pulse," IEEE Photon. Technol. Lett., vol. 20, pp. 1869-1871, 2008.

[18] D. N. Papadopoulos, Y. Zaouter, M. Hanna, F. Druon, E. Mottay, E. Cormier, and P. Georges, "Generation of 63 fs $4.1 \mathrm{MW}$ peak power pulses from a parabolic fiber amplifier operated beyond the gain bandwith limit," Opt. Lett., vol. 32, pp. 2520-2522, 2007.

[19] T. Schreiber, C. K. Nielsen, B. Ortac, J. P. Limpert, and A. Tünnermann, "Microjoule-level allpolarization-maintaining femtosecond fiber source," Opt. Lett., vol. 31, pp. 574-576, 2006.

[20] C. Finot and G. Millot, "Synthesis of optical pulses by use of similaritons," Opt. Express, vol. 12, pp. 51045109, 2004.

[21] C. Finot, S. Pitois, and G. Millot, "Regenerative 40-Gb/s wavelength converter based on similariton generation," Opt. Lett., vol. 30, pp. 1776-1778, 2005. 